\title{
Diagnóstico municipal de la violencia y la criminalidad en Jundiaí, São Paulo
}

\author{
Cristina Neme, M en C Soc.(I)
}

\begin{abstract}
Neme C.
Diagnóstico municipal de la violencia y la criminalidad en Jundiaí, São Paulo. Salud Publica Mex 2008;50 supl I:SI 9-S28.
\end{abstract}

\begin{abstract}
Resumen
Objetivo. Este artículo presenta los resultados del diagnóstico de la violencia y la criminalidad en el municipio de Jundiaí, São Paulo. Material y métodos. Se han analizado los datos oficiales relacionados con la mortalidad externa durante el periodo 1997-2003 y los crímenes para el de 200 I-2005 en dicho municipio; el objetivo es examinar su magnitud y distribución en términos de población y espacio. Se aplicó una encuesta de opinión y se analizaron datos cualitativos que suministraron grupos focales de la población y profesionales de la seguridad pública. Resultados. Se ha identificado que las principales causas de mortalidad violenta en el municipio son los accidentes de tránsito, seguidos por los homicidios, y que las víctimas son en una proporción mayoritaria jóvenes del sexo masculino. Conclusiones. Las fuentes oficiales ofrecen limitaciones a la elaboración de un diagnóstico más preciso, ya que las percepciones de la población respecto de ciertos problemas, sobre todo el tráfico de drogas, la violencia en las escuelas y los crímenes contra el patrimonio, revelan una notificación insuficiente o incluso inexistencia de datos consistentes acerca de estos fenómenos.
\end{abstract}

Palabras clave: crimen; violencia; diagnóstico; seguridad; Jundiaí, São Paulo; Brasil

\section{Neme C.}

Municipal diagnosis of violence

and criminality in Jundiaí - São Paulo.

Salud Publica Mex 2008;50 suppl I:S I9-S28.

\begin{abstract}
Objective. This article presents the results of a violence and criminality diagnosis in the municipality of Jundiaí, São Paulo. Material and Methods. Official data on external mortality for the duration of the 1997-2003 period were analysed as well as criminal occurrences in the municipality during the 200I-2005 period in order to estimate their magnitude and distribution both across the population and on space.An opinion poll was carried out and qualitative data were produced through focus groups with the population and public security professionals. Results. It was verified that traffic accidents are the main cause of violent mortality in the municipality followed by homicides. It was further established that the victims are mainly young males. Conclusions. Additionally, official sources present limitations to the elaboration of a deepened analysis given that popular perceptions of specific problems, particularly drugs traffic, school violence and crimes against property reveal under-notification or even inexistence of consistent data on these phenomena.
\end{abstract}

Key words: crime; violence; diagnosis; safety; Jundiaí, São Paulo; Brazil

(I) Núcleo de Estudios de la Violencia, Universidad de São Paulo. São Paulo, Brasil.

Fecha de recibido: 26 de abril de 2007 - Fecha de aceptado: 29 de enero de 2008

Solicitud de sobretiros: Cristina Neme. Núcleo de Estudios de la Violencia, Universidad de São Paulo. São Paulo, Brasil.

Av. Profesor Lúcio Mortins Rodríguez, Travesa 4, Bloco 2-Cidade Universitária, Sao Paulo-SP 05508-900 Brasil

Correo electrónico: crisneme@usp.br 
$\mathrm{L}^{2}$ a violencia en Brasil, en particular la criminal, representa una cuestión nacional y ocupa un puesto central en el debate público y la arena política a partir de la década de 1980. Los estudios enfocados en la gravedad de la violencia en el país se han basado sobre todo en la evolución de las tasas de homicidio y han revelado el aumento expresivo de esas prevalencias a lo largo del tiempo (1980-2000) y su desigual distribución entre la población. De modo general, dichos estudios indican que las tasas se elevan conforme se avanza del centro a la periferia de las grandes ciudades y según se clasifiquen las víctimas por edad o género; en este sentido, son los jóvenes del sexo masculino y los habitantes de las áreas periféricas de las capitales o grandes ciudades las principales víctimas del homicidio. ${ }^{1}$ Debe señalarse que los homicidios constituyen la única base nacional de datos sobre violencia que permite hacer comparaciones confiables entre diferentes regiones del país ${ }^{2}$ y parecen representar el ápice de las diversas formas de violencia que afligen a la sociedad brasileña.

A mediados del decenio de 1990, el gobierno federal propuso directrices nacionales en el campo de la seguridad a fin de orientar y articular estados y municipios en la creación de políticas de seguridad más racionales y consistentes; se incentivaron así la producción y la sistematización de datos y la elaboración de diagnósticos para subvencionarlas. En fecha más reciente, el Ministerio de Justicia, por medio de la Secretaría Nacional de Seguridad Pública, y de acuerdo con las orientaciones del Plan Nacional de Seguridad Pública, ${ }^{3}$ ha promovido en los municipios la participación activa en la difusión de la seguridad; para ello ha condicionado la concesión de recursos federales a la formulación de diagnósticos y planes municipales de seguridad. Esta iniciativa dispone que se confiera un tratamiento más racional a la cuestión de la inseguridad pública, muchas veces descuidada por los gobiernos, y se incorpore a las municipalidades en una temática que tradicionalmente ha estado limitada a los gobiernos estatales, toda vez que las dos principales fuerzas policiales del país se encuentran subordinadas de manera directa a esos gobiernos.

En tal contexto, el Núcleo de Estudios de Violencia de la Universidad de São Paulo (NEV/USP) elaboró en 2006 un diagnóstico de la violencia y la criminalidad y un plan municipal de seguridad a solicitud del ayuntamiento de Jundiaí, una ciudad mediana, ubicada a cincuenta kilómetros de São Paulo. La finalidad fue identificar la dinámica de la violencia y la criminalidad en la ciudad, además de proponer la articulación y la integración de diferentes niveles de gobierno, instituciones y organizaciones sociales en la promoción de políticas públicas de prevención de la violencia por medio del Plan Municipal de Seguridad.
Este artículo tiene como objetivo presentar los resultados del diagnóstico de la violencia y la criminalidad en Jundiaí, con vistas a la cuestión de la seguridad en el municipio. Se entiende seguridad como un estado en el cual los riesgos de sufrir daños físicos, psicológicos o materiales se controlan de tal modo que se preserven la salud y el bienestar de los individuos y la comunidad. La seguridad se considera en sus dos dimensiones: la objetiva, que puede verificarse en función de patrones conductuales y ambientales objetivos, y la subjetiva, definida en relación con el sentimiento de seguridad o inseguridad de la población. ${ }^{4}$ En este estudio no se alcanzan todos lo fenómenos implícitos en dicha definición. Se consideran los principales fenómenos violentos, de naturaleza criminal y no criminal, así como los principales tipos delictivos, que implican o no violencia, y que afectan la seguridad de las personas y la comunidad. Se busca conocer la magnitud y la distribución de la violencia y la criminalidad en el municipio y la población, de acuerdo con las fuentes oficiales, así como la victimización y la identificación de los mayores problemas de inseguridad del municipio, ya desde la perspectiva de la población, ya desde la óptica de los profesionales que actúan en el área de la seguridad. El objetivo es comparar los datos obtenidos a partir de los registros oficiales con las percepciones de seguridad/inseguridad.

Frente a la tradicional inconsistencia de la planificación en el área de la seguridad pública en Brasil y la enajenación de los municipios en relación con las políticas de seguridad, la elaboración de diagnósticos locales que subvencionen planes locales de seguridad a partir de la iniciativa de los ayuntamientos emerge como una oportunidad de crear una base de datos en el municipio que permita planificar, vigilar y evaluar las políticas en el campo de la prevención y la seguridad. El programa también incluye la incorporación de las autoridades locales y la comunidad en la problemática de la seguridad, dado que la elaboración del diagnóstico y el plan deben ocurrir de forma participativa. Hay que observar que la operación del plan propuesto, bajo la dirección del ayuntamiento, no se halla en manos del equipo del Núcleo de Estudios de la Violencia y por lo tanto no se refiere en este artículo.*

\footnotetext{
* El proyecto Diagnóstico de la Violencia y Plan Municipal de Seguridad de Jundiaí lo desarrolló el siguiente equipo de investigadores del Núcleo de Estudios de la Violencia: Cristina Neme, Paula Ballesteros, Bruna Charifker, Gabriela Toledo Silva y Paulo de Mesquita Neto. El trabajo se limitó a la elaboración del diagnóstico y el plan.
} 


\section{Material y métodos}

El presente estudio se basa en el análisis de un conjunto de datos cuantitativos y cualitativos del municipio de Jundiaí. Para la obtención de datos cuantitativos se han utilizado fuentes del área de la salud y la seguridad pública. El Sistema de Datos sobre Mortalidad* ha permitido identificar las dimensiones y la distribución de la mortalidad violenta en el municipio (las principales causas y el perfil de las víctimas) durante el periodo 1997-2003. A partir de los datos sobre mortalidad por causas externas originados en el área de la salud, con base en la Clasificación Internacional de Enfermedades (CID-10), se han seleccionado las siguientes variables: muertes por agresión (homicidios), accidentes (de tránsito y con arma de fuego), suicidios, muertes por acción de la policía y actos cuya intención no se determinó en ese lapso. Se ha realizado un análisis de serie temporal descriptiva para el periodo y se han calculado la mortalidad proporcional y las tasas de mortalidad (por 100000 habitantes) al año para la población total, por género y edades. El Sistema Estatal de Recopilación de Estadísticas Criminales ${ }^{5}$ y el Banco de Datos de la Comisaría Seccional de Policía de Jundiaí permitieron identificar, respectivamente, la magnitud de los delitos durante el periodo 2001-2005 y su distribución por barrios en 2005. A partir de las estadísticas criminales se han cuantificado datos acerca de crímenes violentos y no violentos, como homicidios, intentos de homicidio, robos seguidos de muerte, lesiones corporales, crímenes sexuales, secuestros, robos, hurtos y tráfico de drogas, además de infracciones cometidas por adolescentes, durante 2001 a 2005. Se llevó a cabo un análisis de serie temporal descriptiva para el periodo y se calcularon la proporción y las tasas (por 100000 habitantes) de los delitos al año. Sólo para 2005 fue posible mapear algunos crímenes por barrios de la ciudad.

Como fuentes oficiales alternativas de datos sobre infracciones cometidas por adolescentes y violencia sexual se han utilizado dos municipales: el Programa Señal Amarilla (Secretaría Municipal de Integración Social) y el Programa Integral de Asistencia a las Víctimas de Violencia Sexual (Secretaría Municipal de Salud), cuyos datos complementaron los registros policiales con informaciones sobre la naturaleza de los crímenes y el perfil de víctimas y perpetradores. Otra fuente para reconocer el móvil de los casos de homicidio e intento de homicidio es el archivo de Denuncias (petición inicial del

\footnotetext{
* Secretaría de Vigilancia en Salud, Ministério da Saúde, Brasil. Sistema de Información sobre Mortalidad 2005.
}

proceso criminal) elaboradas por el promotor de justicia local; así se obtuvieron informaciones sobre los casos denunciados por el ministerio público de 2003 a 2005.

Una encuesta de opinión sobre las percepciones de la población respecto de la violencia se aplicó a 301 ciudadanos con el objetivo de identificar el sentimiento de inseguridad de la población y su exposición a la violencia (victimizaciones directa e indirecta). El cuestionario lo ideó el NEV/USP y la encuesta la aplicó el Instituto Brasileño de Opinión Pública y Estadística en el municipio de Jundiaí entre el 14 y el 17 de enero de $2006 .^{*}$

En términos cualitativos, se han recopilado datos por medio de grupos focales conformados por profesionales participantes en el área de seguridad y representantes de la sociedad civil, y se obtuvieron respectivamente en los meses de marzo y abril de 2006.

Con los profesionales de la seguridad se han integrado tres grupos focales: el primero reunió a 10 policías civiles, ocho hombres y dos mujeres, cuyo tiempo de trabajo oscilaba entre 13 y 28 años. El segundo grupo incluyó a 10 policías militares, cinco mujeres y cinco hombres, cuyo tiempo de trabajo variaba de 8 a 27 años. El tercer grupo conjuntó a 20 guardias municipales, $\neq$ 18 hombres y dos mujeres, casi todos con tiempo de trabajo de 10 a 20 años. Los participantes se eligieron por medio de designación, a cargo de los respectivos comandantes, policiales y guardias que representaran a distintos sectores de las corporaciones y actuaran en diferentes áreas de la ciudad. Las discusiones, grabadas y taquigrafiadas se orientaron de acuerdo con un guión centrado en tres puntos: a) seguridad/inseguridad en la ciudad, b) relación entre las corporaciones policiales y la población y c) vínculo entre las propias corporaciones.

Asimismo, se ha integrado un grupo focal con 10

\footnotetext{
* El universo de la encuesta es la población de 16 años o más del municipio y el modelo de muestra utilizado es el de conglomerados en dos etapas. En la primera etapa se seleccionan los conglomerados: sectores censatarios, con PPT (probabilidad proporcional al tamaño) sistemático. La medida de tamaño es la población de 16 años o más residente en los sectores. En la segunda etapa se selecciona de cada conglomerado un número fijo de electores de acuerdo con las cuotas de las siguientes variables: sexo, edad, instrucción y actividad económica. El intervalo de confianza previsto es de $95 \%$ y el margen de error máximo calculado es de seis puntos porcentuales hacia arriba o abajo sobre los resultados encontrados en el total de la muestra. Hubo filtración de todos los cuestionarios tras la realización de las entrevistas y fiscalización en casi $20 \%$ de los cuestionarios.

‡ El número de guardias municipales es elevado porque el comandante de la institución presentó un número superior al demandado, interferencia que no puede controlarse si se consideran las relaciones de jerarquía características de esas corporaciones.
} 
representantes de la sociedad civil, en su mayoría representantes de asociaciones de habitantes de distintas regiones de la ciudad, invitados por intermediación del ayuntamiento. La discusión ha versado en torno de una relación de tres aspectos: a) seguridad/inseguridad en la ciudad, b) relación entre la población y las corporaciones policiales y c) prioridades de la seguridad. Al final, se llevó a cabo un análisis de contenido del material suministrado por los grupos focales.

Por último, con la finalidad de precisar el diagnóstico, discutir las prioridades del municipio para el plan de seguridad y ampliar la participación de la sociedad en el proceso, se presentó y discutió una versión preliminar del diagnóstico en dos audiencias organizadas por el ayuntamiento, en las cuales comparecieron autoridades locales municipales y estatales (alrededor de 40 personas, representantes de secretarías municipales y estatales, la guardia municipal, las policías, el poder judicial y el ministerio público) y representantes de la sociedad civil organizada (alrededor de 50 individuos, procedentes de asociaciones de barrio, consejos comunitarios de seguridad y organizaciones no gubernamentales). Tres investigadores registraron las reacciones al diagnóstico preliminar y las informaciones que emergieron en las audiencias.

\section{Resultados}

\section{Perfil de la violencia y la criminalidad de acuerdo con datos oficiales}

De modo general, Jundiaí posee elevados parámetros sociales, un gran nivel de riqueza y desarrollo humano y un índice de desarrollo humano (IDH, 0.857) superior al de los estados de São Paulo y Brasil. Su población es de 344779 habitantes (2005), de los cuales más de 90\% se encuentra en el área urbana.

Respecto del perfil de la violencia y la criminalidad, se ha verificado que los accidentes de tránsito se han convertido en la principal causa externa de mortalidad en el municipio durante el periodo de 1997 a 2003, excepto en 2001, cuando los homicidios fueron la primera causa de mortalidad. En ese año, los homicidios representaron $37 \%$ de las muertes por causas externas; en los demás, los accidentes de tránsito ocasionaron 31 a 50\% de los decesos. Tanto los suicidios como los accidentes con arma de fuego correspondieron a un promedio de $5 \%$ de las muertes a lo largo de ese lapso. No se han registrado muertes por acción de la policía y ha sido notoria la gran proporción de muertes cuya intención no pudo determinarse: 37\% en 2000 (figura 1).

Durante esos años, la tasa de mortalidad por accidentes osciló entre el mínimo de 19.4 y el máximo de 34.1 muertes por 100000 habitantes. Las víctimas fatales de los accidentes son en su mayoría del sexo masculino (una proporción que ha variado de 70 a $86 \%$ en el periodo), situadas sobre todo en las edades de 20 a 29 años, seguidas por las de 30 a 39 años.*

Los homicidios constituyen la segunda causa de mortalidad violenta en Jundiaí, lo cual supone un promedio de 29\% de las muertes en esos siete años. En 2001

* Sistema de Información sobre Mortalidad 2005.

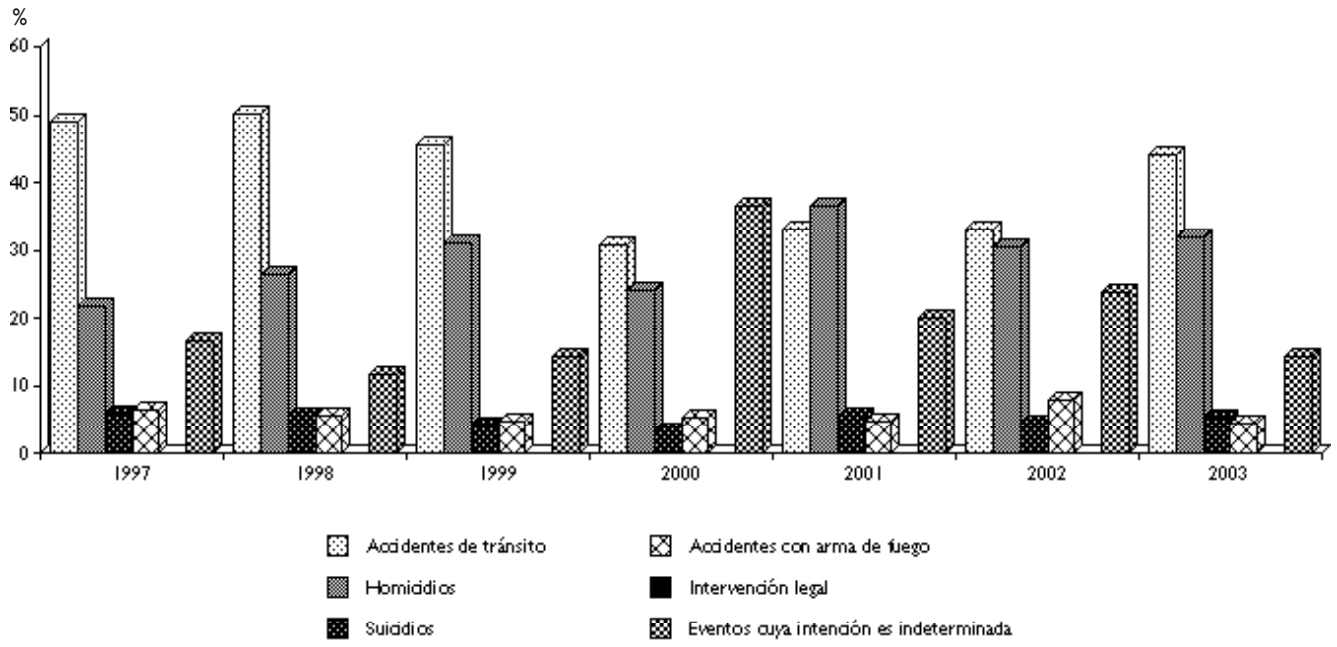

Fuente: Sistema de Información sobre Mortalidad, 2005

Figura I. Proporción de mortalidad por causas externas 1997-2003, Jundiaí, Brasil 
se alcanzó el mayor número de muertes por homicidio, con 84 víctimas (25.6 muertes por 100000 habitantes); luego de ese año se inició una tendencia decreciente de esa cifra, también verificada en el promedio del estado de São Paulo (figura 2).

El arma de fuego ha sido el principal medio utilizado en los crímenes (71\% de los casos en 2003) y la víctima de homicidio es casi siempre del sexo masculino (más de 90\%) y joven. Entre los individuos de 20 a 29 años y los adolescentes de 15 a 19 años se presentan las mayores tasas de mortalidad por 100000 habitantes. En 2003, dichas tasas correspondieron a 46.3 y 50.9 , respectivamente. Si se considera a los jóvenes del sexo masculino de 20 a 29 años, la tasa se eleva a 89 por 100000 (figura 3).

Por último, se ha reconocido que los homicidios se distribuyeron en grupos de barrios con diferentes características socioeconómicas. Si se observan en conjunto los barrios de baja renta y los barrios aislados, se advierte que la mitad de los homicidios ocurrió en tales localidades y la otra mitad en barrios de media y alta renta.* $^{*}$

El municipio posee una tasa de lesiones corporales superior a la de la capital (410 y 310 casos por 100000 habitantes en 2004, respectivamente). Las lesiones corporales tienen frecuencia mayor entre las mujeres: de los 1553 episodios de 2005, 57\% se registró en la Comisaría de Defensa de la Mujer y, por lo tanto, se refiere a víctimas del género femenino ( $43 \%$ se distribuye en las otras siete comisarías policiales del municipio, sin información disponible sobre el sexo de la víctima). ${ }^{\ddagger}$ Se ha identificado que las amenazas a la integridad física contra las mujeres registradas en la Comisaría de Defensa de la Mujer local se acompañan de lesiones corporales (figura 4).

La víctima de violencia sexual también muestra un predominio femenino. Si se suman los principales

\footnotetext{
* Se consideran barrios de alta renta aquellos con concentración (más de $30 \%$ ) de jefes de domicilio cuya renta es superior a 10 sueldos mínimos (actualmente $\mathrm{R} \$ 380$ ); barrios de baja renta son los que tienen concentración de jefes de domicilio con renta hasta de tres sueldos mínimos; en los barrios de renta media la proporción de jefes de domicilio con renta es mayor de 10 (menos de tres sueldos no alcanza el promedio de la ciudad). Los barrios aislados son suburbios nuevos que se formaron en áreas periféricas de la ciudad y para los cuales no existían datos socioeconómicos. Fuente: Censo 2000, Instituto Brasileiro de Geografia e Estatística.

‡ Sistema Estadual de Coleta de Estatísticas Criminais, 2006.

\$ Según el código penal brasileño, coaccionar a la mujer a la conjunción carnal, mediante violencia o grave amenaza, constituye un crimen de violación. Obligar a alguien, con violencia o grave amenaza, a practicar o permitir que se practiquen actos de naturaleza sexual, diferentes de la conjunción carnal (p. ej., sexo oral o anal), representa un atentado violento al pudor.
}

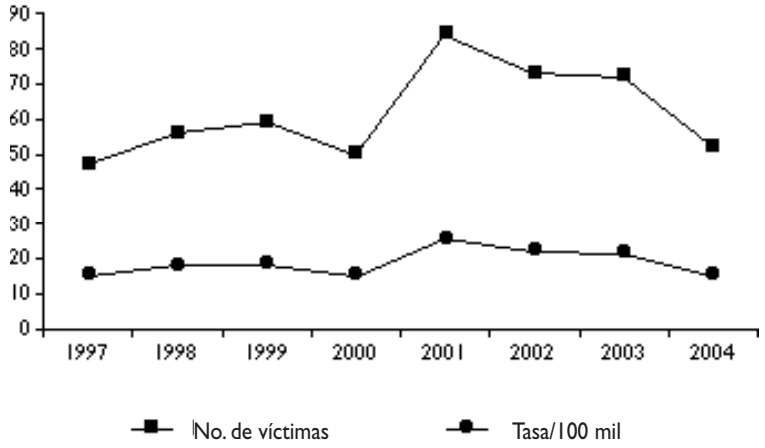

Fuente: Sistema de Información sobre Mortalidad, 2005

Figura 2. Número y tASA de homicidios 1997-2004, JUNDIAÍ, BRASIL

crímenes sexuales (violaciones y atentados violentos al pudor), $\$$ se obtiene el promedio anual de 58 casos registrados por la policía en el periodo 2001-2005.* Por otro lado, si se toman los datos del Programa Integral de Asistencia a las Víctimas de Violencia Sexual se observa que éste ha atendido 316 casos de violencia sexual entre 2000 y 2005, de los cuales la violación y el atentado violento al pudor representaron 83.6 por ciento. De acuerdo con los datos del programa, $90 \%$ de las víctimas corresponde a mujeres, $69 \%$ a niños y adolescentes y, en la mayoría de los casos $(67 \%)$, los agresores son allegados a la víctima y pertenecen a su círculo familiar (padre, padrastro, tío, abuelo, novio, entre otros), la vecindad y los amigos cercanos. ${ }^{\ddagger}$

El crimen de extorsión mediante secuestro se ha incrementado en grado significativo en el periodo estudiado, desde dos casos registrados en 2001 hasta ocho en 2005.*

Jundiaí muestra tasas elevadas y crecientes de crímenes contra el patrimonio: de 2001 a 2005 los robos aumentaron $25 \%$ y los hurtos $39 \%$; además, la tasa de hurtos ( 1505 por 100000 habitantes en 2005) fue superior a la de la capital y el promedio del estado. Es también considerable la incidencia de robo y hurto de vehículos, mayor al promedio del estado y los municipios de la región, aunque menor al de la capital. ${ }^{*}$ Los crímenes contra el patrimonio (robos y hurtos en general) se concentran en barrios centrales de renta más alta. $\$$

De acuerdo con los registros oficiales, los delitos

\footnotetext{
* Sistema Estatal de Recopilación de Estadísticas Criminales, 2006.

¥ Secretaría Municipal Salud de Jundiaí, Clínica de Salud de la Mujer. Programa Integral de Asistencia a las Víctimas de Violencia Sexual, 2006.

§ Delegación Seccional de Policía de Juniaí, 2006.
} 


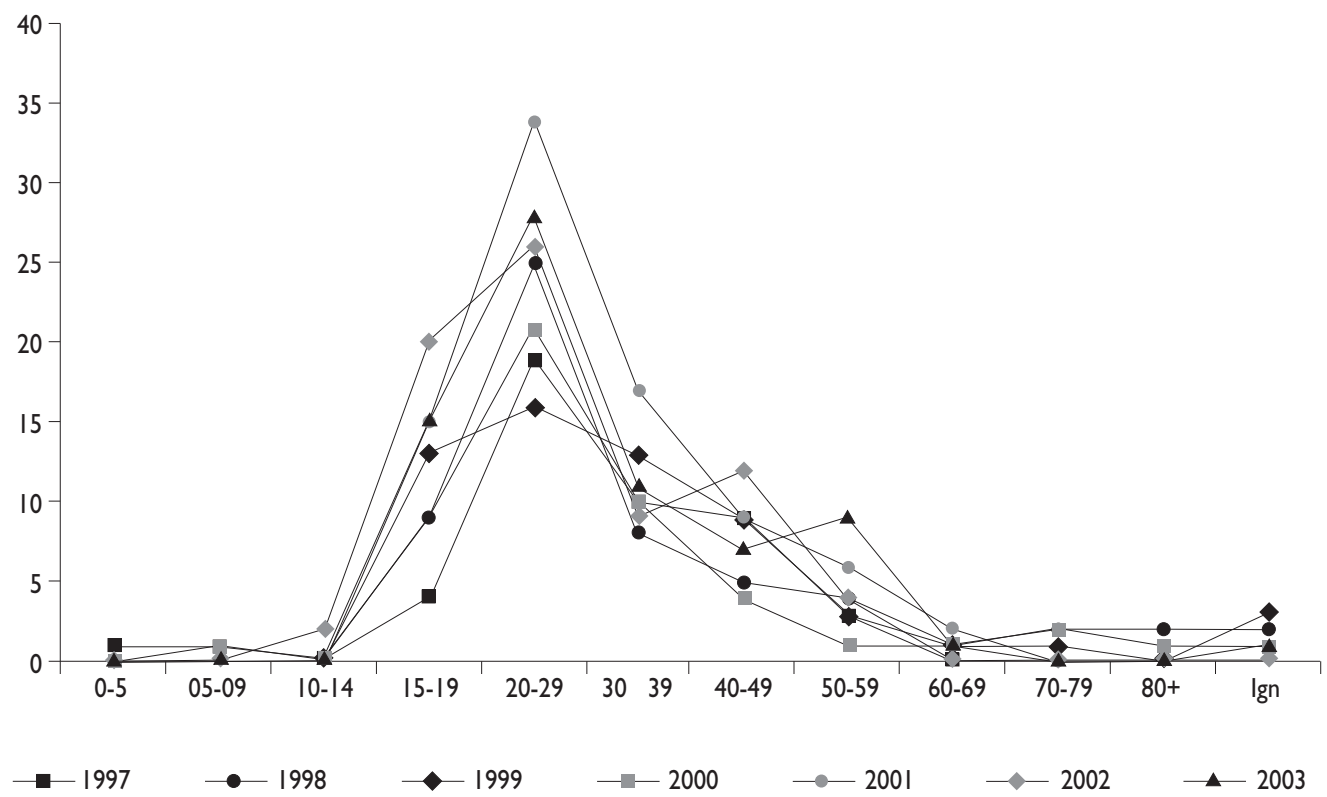

Fuente: Sistema de Información sobre Mortalidad, 2005

Figura 3. Número de lesiones corporales y amenazas contra mujeres 200I-2005, Jundiaí, Brasil

de tráfico aumentaron de manera notable entre 2001 y 2005 (104\%).* De los participantes en los delitos de tráfico registrados por la policía en 2004, sobresalen los adolescentes y jóvenes del sexo masculino: $38.6 \%$ tenía edades de 14 a 17 años, seguido por $24.2 \%$ en los límites de 18 a 20 años y $16.4 \%$ en el intervalo de 21 a 25 años.* El tráfico de drogas es la principal infracción cometida por adolescentes que satisfacen una medida socioeducativa, es decir, es el principal motivo de aprehensión de adolescentes, en su gran mayoría del sexo masculino (más de $90 \%$ ).

\section{Percepciones relacionadas con la seguridad}

En términos cualitativos, cuando se consideran las

* Delegación Seccional de Policía de Jundiaí/Delegación de Investigación sobre Estupefacientes, 2006.

‡ Secretaria Municipal de Integración Social de Jundiaí, Programa Señal Amarilla. Es el programa municipal de acompañamiento de los adolescentes en conflicto con la ley que cumplen medidas socioeducativas, diferentes de la internación, en el municipio. Hasta entonces no había unidad de internación local y los adolescentes que cumplían esa medida se transferían a las unidades de la capital (São Paulo). No se obtuvo información sobre los adolescentes internados.

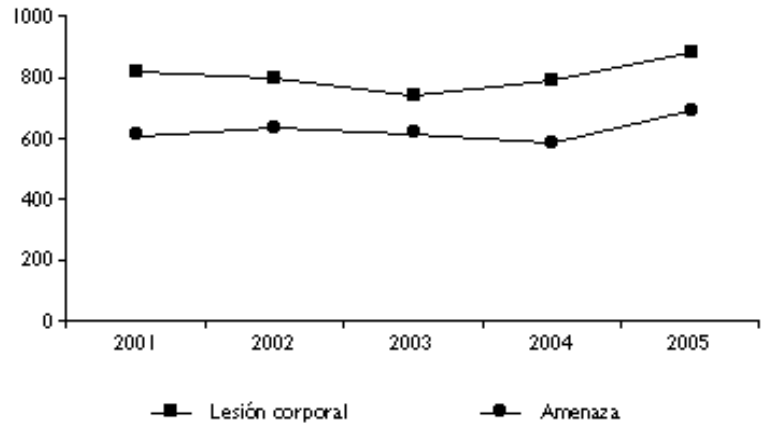

Fuente: Sistema Estatal de Estadísticas Criminales y Delegación de Defensa de la Mujer de Jundiaí, 2006

Figura 4. Número de LESIONES CORPorales y AMENAZAS CONTRA MUJeres 200I-2005, JUNDIAí, BRASIL

percepciones de la población, liderazgos comunitarios y agentes de seguridad respecto de la seguridad en el municipio, se destacan dos problemas: el incremento del consumo y el tráfico de drogas y la inseguridad en las escuelas públicas.

Esta última está considerada un problema relevan- 
te, ya que puede alterar el acceso y la asistencia de los alumnos a las escuelas. En esencia, se teme la adicción y el tráfico de drogas en el entorno escolar e incluso su penetración en las unidades, así como los riesgos del contacto de los alumnos con consumidores o traficantes en los horarios de entrada y salida de las escuelas. La proximidad de los bares, que comercializan bebidas alcohólicas, también es fuente de inseguridad en las escuelas y, por otro lado, la dificultad de control disciplinario de las propias escuelas sobre los alumnos también ha favorecido la sensación de inseguridad. En la perspectiva de una población que relaciona inseguridad con evasión escolar, la falta de control policial en el entorno escolar, sobre todo en los horarios de entrada y salida de los alumnos en las escuelas, es un grave problema aún no enfrentado por las autoridades.

Se reconocen el consumo y el tráfico de drogas de forma acentuada en diferentes barrios de la ciudad e incluso en el interior de las escuelas, con afectación de los adolescentes y los jóvenes. Dichos crímenes se vinculan con la ocurrencia de otras infracciones (robo, hurto, homicidios, lesiones y violencia familiar) y también con la situación de vulnerabilidad de los jóvenes que carecen de ocupación en las áreas periféricas desprovistas de centros de deporte y ocio atractivos. Se percibe la actuación policial en relación con el problema como negligente o ineficaz.

Se ha señalado que la ausencia de control policial (policías o guardias municipales) en los barrios periféricos es factor de inseguridad, así como la falta de iluminación pública. Los crímenes contra el patrimonio, como robos y hurtos, también afectan a la población de esos suburbios donde el recurso de la seguridad privada, aunque creciente, todavía no se ha traducido en garantías de seguridad.

Se han ratificado tales percepciones con la encuesta de opinión sobre el sentimiento de inseguridad y exposición a la violencia. En cuanto a esta última, destacan los atracos en el barrio, un crimen que ha presenciado $19.3 \%$ de la población y lo ha oído mencionar $32.9 \%$ (en los últimos tres meses), aunque menos de $2 \%$ ha sufrido robo con amenaza armada (en los últimos 12 meses); de igual modo, una proporción de $47.2 \%$ ha visto en la calle el consumo de drogas (en los últimos tres meses). Además, $17.9 \%$ ha observado a una persona agredida (en los últimos tres meses), $11.3 \%$ ha sido testigo de amenaza con cuchillo a una persona y también de tiroteos (en los últimos tres meses) y $7.6 \%$ ha padecido o presenciado una amenaza de muerte (en los últimos tres meses).

De la evaluación de los habitantes respecto de la situación de su barrio, sobresalen algunos factores de inseguridad que ocurrieron con bastante frecuencia, como los grafitos en muros o casas (47.5\%), ingestión de alcohol (46.8\%) y consumo de drogas (43.9\%) en locales públicos. Una proporción de 28 a $35.9 \%$ considera que los crímenes, como la venta de drogas en locales públicos o el robo de coches y residencias, ocurren con bastante frecuencia. La provocación consecutiva a la ingestión de bebidas alcohólicas y el consumo y venta de drogas se destacan como causas de violencia, lo cual puede observarse también entre alumnos y en traficantes en las puertas de las escuelas, lo que constituye asimismo un factor de violencia para la mayoría de los entrevistados.

Los policías son quienes delinean el problema de la violencia doméstica, ya que los conflictos interpersonales, peleas entre vecinos, reyertas familiares por consumo de drogas y violencia contra mujeres ocupan gran parte de su trabajo cotidiano. En su percepción, dichas demandas recurrentes, cuya naturaleza es social y no propiamente criminal, no las absorbe una red de instituciones que deberían responder al problema, y terminan por entorpecer o sobrecargar el trabajo policial. En cuanto a la percepción de la población en relación con el problema, la encuesta de opinión ofrece algunas indicaciones: $22 \%$ considera que la agresión física de parejas frente a los hijos ocurre con frecuencia, así como las discusiones o las agresiones verbales ( $33 \%)$. Se observa además una mayor propensión de los padres a golpear a sus hijos en los casos de hurtos, consumo de drogas (marihuana) y trazo de grafitos en los muros (alrededor de 10\%); en cambio, en relación con ciertos comportamientos, como mentir, faltar o crear problemas en las clases, tal tendencia no alcanza 2 por ciento.

\section{Discusión}

A continuación se discuten los principales resultados del diagnóstico y las limitaciones de las fuentes oficiales utilizadas en su elaboración. En primer lugar, es necesario señalar que la notificación insuficiente de los crímenes se debe a problemas de procedimiento de los propios sistemas de registro o a la resistencia de la población a denunciar la falta en las comisarías de policía. El porcentaje de denuncia es variable, según sean el tipo de crimen y la metodología aplicada en encuestas de victimización, pero se considera que es de $70 \%$, de acuerdo con distintas encuestas realizadas entre 1988 y 2002 en Brasil. ${ }^{6}$ En segundo lugar, hay que recordar que, para el caso de ciertos crímenes, las estadísticas criminales pueden reflejar también la mayor o menor actuación de la policía, y no siempre el aumento o la reducción de la criminalidad.

El Sistema de Datos sobre Mortalidad es la única base nacional de mortalidad violenta y suministra en esencia información relacionada con la frecuencia de 
los episodios y el perfil de las víctimas inferidos de las declaraciones del óbito. Sin embargo, es importante resaltar que los datos sobre mortalidad provocada por agentes legales no se consignan en este sistema, incluso si tales datos son del dominio estatal, y que la mortalidad por causa indeterminada es elevada; en consecuencia, las muertes por homicidio $u$ otras causas violentas pueden estar omitidas. El Sistema Estatal de Recopilación de Estadísticas Criminales ofrece datos sobre el tipo y la frecuencia de los crímenes, provenientes de los boletines registrados en las comisarías de policía, pero no discrimina su distribución por barrios en la ciudad ni tampoco el perfil de agresores y víctimas.

En relación con los homicidios, la comparación entre las dos fuentes incrementa la confiabilidad de la información. Para el municipio de Jundiaí, se observa que las dos fuentes muestran la misma tendencia decreciente para los homicidios, aunque el número de víctimas consignado a lo largo del periodo por los registros criminales es inferior al de la fuente de la salud. Sólo en 2004 coincide la información de ambas fuentes.*

Sin duda, representa una dificultad obtener mayores informaciones acerca del perfil de la víctima, además del sexo y la edad, y son raras las fuentes que recogen datos del perfil del agresor. Ha sido posible delinear una noción parcial de los factores que llevan a la perpetración de los crímenes contra la vida ocurridos en el municipio a partir de las denuncias del organismo promotor criminal local. Se ha verificado que de los 115 casos de homicidios e intentos denunciados ante el ministerio público en el periodo 2003-2005, la gran mayoría $(80 \%)$ tuvo como origen algún tipo de pelea (venganzas, peleas familiares o disputas por deudas). Pese a ello, hay un conjunto equivalente de casos archivados, cuya autoría y motivo se desconocen. ${ }^{\ddagger}$ Además, como se ha observado, los homicidios no ocurren en contextos homogéneos, lo que refuerza la necesidad de efectuar estudios locales más minuciosos para que se comprenda la dinámica de ese crimen.

Los casos de "secuestros relámpago" indican que no todos los crímenes figuran en las estadísticas estatales, probablemente a causa de la falta de un patrón en la clasificación policiaca de esa modalidad reciente de crimen, en la cual se secuestra a la persona de manera

\footnotetext{
* Para este año, el dato del Sistema de Datos sobre Mortalidad se obtuvo de forma preliminar. Se advierte que, en los registros criminales, las muertes están clasificadas según distintas categorías jurídicas, de forma que se han concertado las clasificaciones para posibilitar una comparación entre las diferentes fuentes.

‡ Promotoría de Justicia Criminal de Jundiaí/Ministério Público del Estado de São Paulo. Elaboración: NEV/USP, 2006.
}

momentánea mientras se sustraen los valores de su propia víctima o sus familiares en cajeros automáticos bancarios. Entre 2004 y 2005 se registraron 18 casos en la Comisaría Seccional de Policía de Jundiaí, una información que entonces no constaba en esos términos en el Sistema Estatal de Recopilación de Estadísticas Criminales, aunque dicho crimen provoque una gran inseguridad en la población.

En cuanto a los niños, destaca el hecho de que el Consejo Tutelar no tiene datos sistematizados sobre la atención realizada y, en consecuencia, no vigila la violencia contra los niños en el municipio. Es el Programa Integral de Asistencia a las Víctimas de Violencia Sexual el que proporciona mayores informaciones sobre violencia contra los niños y adolescentes. De los registros criminales se obtiene sólo la frecuencia de los casos de malos tratos (negligencia) en contra de los niños y adolescentes y la información de que se cometen sobre todo en residencias ( $85 \%$ en 2005).*

No se consiguieron datos oficiales más detallados en relación con la violencia doméstica, aunque el problema se destaque en la experiencia policial. Las fuentes utilizadas ofrecen informaciones sobre violencia contra mujeres, niños y adolescentes y otros delitos vinculados con la violencia doméstica, pero sus registros no permiten separar los datos de tal forma que pueda verificarse el alcance del fenómeno. Por ejemplo, la violencia contra ancianos se incluye en "otros delitos" en la Comisaría de Defensa de la Mujer, una dependencia especializada que prioriza los registros sobre violencia contra la mujer.

Por su parte, los resultados del diagnóstico muestran que si bien los accidentes de tránsito son la principal causa de mortalidad violenta en el municipio, la población no percibe el tránsito como fuente de inseguridad. Como se sabe, existe un sólido nexo entre el consumo de alcohol y los accidentes de tránsito, ${ }^{7}$ pero sólo los policías intervienen en los numerosos casos de carreras de coches no autorizadas ${ }^{*}$ por la noche y en los fines de semana, cuando tienen lugar peleas y accidentes, sin contar con el hecho de que muchos accidentes se relacionan con el consumo de bebidas alcohólicas.

El municipio se distingue de las capitales y ciudades grandes y medianas brasileñas, que poseen tasas de homicidio elevadas: su incidencia de 9.5 asesinatos por 100000 habitantes (2005) es más baja que las tasas del estado de São Paulo y la capital, que en el mismo año

\footnotetext{
* Delegación Seccional de Policía de Jundiaí, 2006.

‡ Las carreras de coche ilegales (rachas) son disputas entre jóvenes en las que se realizan maniobras arriesgadas a grandes velocidades.

§ Sistema Estatal de Recopilación de Estadísticas Criminales, 2006.
} 
correspondieron a 18.2 y 24 , respectivamente. $\$$ Pese a ello, cabe observar que los homicidios aún representan un grave problema nacional, no obstante la tendencia decreciente verificada a partir de 2000 tras décadas de crecimiento vertiginoso, y representan en Jundiaí la segunda causa de mortalidad violenta.

Es contrastante la preocupación popular por el consumo y tráfico de drogas y la violencia en las escuelas, por un lado, y la ausencia o insuficiencia de informaciones sistematizadas sobre tales fenómenos, por otro. La concentración del tráfico de drogas en pocos barrios, según los datos oficiales, no corresponde a la percepción de la población, que reconoce el problema en barrios diversos. Tan sólo las denuncias efectuadas en el servicio de Llame y denuncie durante sus primeros seis meses de funcionamiento (julio a diciembre de 2005) rebasan el número de casos de tráfico registrados por la policía durante todo el año. Son 476 denuncias de tráfico de estupefacientes, las cuales correspondieron a $50 \%$ del total de las denuncias recibidas por el servicio telefónico.*

La preocupación con las drogas parece reflejar la gravedad de un problema que es nacional: la expansión del tráfico y la violencia vinculada con el fenómeno. Los jóvenes pueden cooptarse como mano de obra del tráfico y pasar a forma parte entonces de un círculo de criminalidad que difícilmente puede abandonarse. O bien, si al principio los sujetos son sólo consumidores, pueden incurrir después en la criminalidad para obtener recursos y saldar sus deudas, o incluso integrarse a pandillas, como es evidente en el caso de Río de Janeiro. En un contexto de gran exclusión social, violencia y corrupción institucional, muchos jóvenes pobres sufren un proceso de socialización negativa, en la medida en que su identidad se construye a partir de la referencia a los patrones que imponen los criminales locales, que tienen a su disposición armas y dinero. ${ }^{8}$ Pese a la diferencia entre las dos ciudades, no puede soslayarse el temor de reproducir el problema, recurrente en grandes ciudades brasileñas, en urbes cuya calidad de vida es superior, pero que no escapan al elevado patrón de desigualdad social brasileño, como es el caso de Jundiaí.

En cuanto a la violencia en las escuelas, no se dispone de datos sistematizados y calificados en la Secretaría de Educación sobre dichos episodios, sólo el número bruto de ocurrencias contra la persona y el patrimonio

\footnotetext{
* Llame y denuncie (Disque-denúncia) de Jundiaí, 2006. Es un servicio de teléfono gratuito y anónimo puesto a la disposición de la población con el objetivo de facilitar e incentivar su participación en la resolución de crímenes.
}

registradas cada año por el conjunto de las escuelas. Para que se detalle el diagnóstico es necesario llevar a cabo una investigación de campo en las escuelas y separar la información oficial existente de los nuevos datos.

Por último, los crímenes contra el patrimonio también son objeto de preocupación de la población. Aunque de acuerdo con los registros oficiales dichos delitos están concentrados en los barrios centrales, en la perspectiva de la población este problema también es recurrente en suburbios periféricos, de tal modo que la notificación insuficiente de robos y hurtos se ha evidenciado al discutir el diagnóstico con la población. Los habitantes de los barrios alejados del centro o periféricos no se reconocen en los indicadores de crímenes contra el patrimonio basados en los registros oficiales y consideran que la falta de denuncia se debe al nulo acceso a las comisarías, ya sea por la distancia física y la deficiente atención, o por el descrédito popular de la efectividad del registro. Los policías, por su parte, reiteran la falta de colaboración de la población, sobre todo en la periferia, y la atribuyen al miedo de sufrir reprensiones (p. ej., al reconocer a los criminales) y la dificultad de desplazarse hasta la comisaría más próxima. La denuncia también puede vincularse con la desconfianza que suscita la policía: en la encuesta de opinión más de $30 \%$ considera que los policías no intervienen en actividades ilícitas (como recibir soborno o proteger traficantes), mientras que $18 \%$ cree que lo hacen con frecuencia. No obstante, en esta cuestión se registra la mayor proporción de los que contestaron no saber o prefirieron no opinar (30\%), lo cual sugiere un notorio recelo.

Los datos oficiales disponibles en la actualidad permiten trazar un primer diagnóstico de la violencia y la criminalidad en la ciudad, pero no reflejan la verdad de los problemas relevantes, como el tráfico de drogas, y no posibilitan la fragmentación de los datos necesaria para detallar mejor el diagnóstico. Además, las instituciones relacionadas con la seguridad (dos policías estatales y una guardia municipal) almacenan sus datos en sistemas no integrados. Es preciso crear un patrón de integración, por lo menos entre instituciones locales que registran datos de naturaleza similar, como las policías estatales (policías civil y militar) y la guardia municipal.

Respecto del poder municipal, cuando es posible obtener algunos datos sobre episodios violentos en sus programas sociales, éstos son datos muy parciales y no siempre están sistematizados. De esa forma, el diagnóstico de la criminalidad y la violencia de Jundiaí apunta hacia la necesidad primera de idear un sistema municipal que centralice la producción de informaciones sobre la violencia y vigile los fenómenos; además, debe proporcionar condiciones para que el municipio sea un actor clave en el área de la seguridad, con énfasis en la prevención. 


\section{Referencias}

I.Waiselfisz J. Mapa da violência III: os jovens do Brasil. Brasília: Organização das Nações Unidas para a Ciência e a Cultura, 2002: 33-34, 48. Peres MFT.Violência por armas de fogo no Brasil. São Paulo: Núcleo de Estudos da Violência, 2004.

2. Lima RK, Misse M, Miranda APM.Violência, criminalidade, segurança pública e justiça criminal no Brasil: uma bibliografia. Rev Bras Infor Bibl Ciên Soc 2000;50:45-I23.

3. Instituto Cidadania. Projeto segurança pública para o Brasil. São Paulo: Instituto Cidadania, 2002.

4. Centre Collaborateur de la Organisation Mondiale de la Santé du Québec pour la Promotion de la Sécurité et la Prévention des Traumatismes, Centre Collaborateur pour la Promotion de la Sécurité dans les Communautés du Institut Karolinska, Organisation Mondiale de la Santé. Sécurité et promotion de la sécurité: aspects conceptuels et opérationnels. Québec, 1998:7-9. Disponible en: http://www.inspq. qc.ca/pdf/publications/149_SecuritePromotion.pdf. [Consultado el 5 de noviembre de 2007].

5. Secretaria de Estado dos Negócios da Segurança Pública de São Paulo, Resolução I60. Sistema Estadual de Coleta de Estatísticas Criminais, 2006. 6. Kahn T. Segurança pública e trabalho policial no Brasil. University of Oxford/Centre for Brazilian Studies, 2003. Disponible en: http://www. brazil.ox.ac.uk/workingpapers/Tulio\%20Khan\%205I.pdf [consultado 5 noviembre 2007].

7. Minayo MCS.A complexidade das relações entre drogas, álcool e violência. Cad Saúde Pública 1998; I4(I):35-42.

8. Zaluar A. Integração perversa: pobreza e tráfico de drogas. Rio de Janeiro: Fundação Getúlio Vargas, 2004. 